\title{
STUDY ON AWARENESS OF SYMPTOMS OF HYPOGLYCAEMIA \& EARLY MANAGEMENT AMONG PATIENTS WITH DIABETES AND THEIR CAREGIVERS
}

\author{
Spoorthi Ashok Pai1, Peter George 2
}

${ }^{1}$ Senior Resident, Department of General Medicine, Father Muller Medical College Hospital, Mangalore, Karnataka, India. 2Professor, Department of General Medicine, Father Muller Medical College Hospital, Mangalore, Karnataka, India.

ABSTRACT

\section{BACKGROUND}

Drug induced hypoglycaemia is common among diabetics on medications. Lack of awareness on hypoglycaemic symptoms among patients and caregivers could pose delay in its identification and treatment. We wanted to evaluate the awareness of symptoms of hypoglycaemia and knowledge on treatment, among diabetics and their caregivers.

\section{METHODS}

This cross-sectional study was done among Type-2 diabetes patients and their caregivers. Diabetics with history of diabetes of over five years and their caregivers, who fulfilled the selection criteria were included. Their knowledge on the symptoms, signs and treatment of hypoglycaemia were compared.

\section{RESULTS}

Among the 200 studied, there were 100 patients and 100 caregivers. Among diabetic patients, 38\% had average awareness about symptoms of hypoglycaemia, whereas $24 \%$ of caregivers were poorly aware. Among the diabetic patients, $40 \%$ had received diabetic education from their clinician. For the management of hypoglycaemia, 38\% patients felt glucose powder or sugar with water as an immediate measure; whereas $39 \%$ caregivers chose water. Majority of patients (60\%) and caregivers (67\%) considered medical help to be the right option for managing hypoglycaemia.

\section{CONCLUSIONS}

Caregivers were poorly aware about symptoms of hypoglycaemia and as the literacy levels of subjects increased, awareness also incresed.

HOW TO CITE THIS ARTICLE: Pai SA, George P. Study on awareness of symptoms of hypoglycaemia \& early management among patients with diabetes and their caregivers. J. Evolution Med. Dent. Sci. 2019;8(16):1262-1264, DOI: 10.14260/jemds/2019/281

\section{BACKGROUND}

Diabetes in India, with over 62 million cases, is emerging as an epidemic. According to Wild et al ${ }^{1}$ people affected with diabetes is predicted to double globally from 171 million in 2000 to 366 million in 2030. In India, diabetes is predicted to affect up to 79.4 million individuals by 2030.2

Hypoglycaemia is a very important and potentially preventable short-term complication occurring in diabetics on drug therapy. ${ }^{3}$ This require immediate medical intervention and is associated with severe neurological or cardiovascular adversities.

Caregivers aware of hypoglycaemic symptoms recognize it faster and initiate adequate measures to prevent morbidity. Lack of awareness on hypoglycaemic symptoms among patients and their caregivers may cause delay in identification and treatment, resulting in poor outcome. We studied the awareness on hypoglycaemic symptoms and knowledge on early management among diabetics and their caregivers.

'Financial or Other Competing Interest': None.

Submission 05-02-2019, Peer Review 14-04-2019,

Acceptance 17-04-2019, Published 22-04-2019.

Corresponding Author:

Dr. Spoorthi Ashok Pai,

Senior Resident,

Department of General Medicine

Father Muller Medical College,

Father Muller Road,

Mangaluru-575002,

Karnataka, India

E-mail: spoorthiashokpai@gmail.com

DOI: $10.14260 /$ jemds/2019/281

\section{(c) $(\$)$}

\section{Aims \& Objectives}

1. To study the awareness of symptoms of hypoglycaemia among patients with diabetes and their caregivers.

2. To study the knowledge on early management of hypoglycaemia among patients with diabetes and their caregivers.

\section{METHODS}

\section{Source of Data}

Diabetic patients and their caregivers attending the outpatient and in-patient services of tertiary teaching Hospital in Southern India.

\section{Study Design}

This was a Cross-sectional study done on Type- 2 diabetes patients and their caregivers over a period of six months.

The study was commenced after obtaining the Institutional Ethics Committee approval. About 200 subjects were included in the study, of which 100 subjects were patients with Type- 2 diabetes for 5 years or more than 5 years and on oral hypoglycaemic drugs or insulin. The remaining 100 were their Caregivers. A caregiver is a person who spends 6 to 8 hours in the same dwelling as the patient, aware of patient's current health issues and treatment. Sample size was taken for convenience. Subjects were selected using Convenience sampling. Patients with diabetes for five or more years and their caregivers, who fulfilled the selection criteria, were included in the study after obtaining a written informed consent. They were administered the questionnaire and asked to mark their responses on the questionnaire form. 
They were provided help, if required, to interpret the questions and to mark their responses. The level of knowledge the patient and the caregiver had were compared. The level of knowledge was also compared with their literacy level.

\section{Inclusion Criteria}

1. Diabetes for 5 years or more than 5 years and on oral hypoglycaemic drugs or insulin.

2. Their caregivers - a person who spends $6-8$ hours in the same dwelling as the patient, aware of patient's current health issues and treatment.

\section{Exclusion Criteria}

1. Patients admitted in intensive care units.

2. Patients or caregivers with neuro-psychiatric illness.

\section{Statistical Methods}

SPSS 23 software was used for data analysis. The data collected was transferred to an Excel data sheet and analysed by frequency, mean, ratios and percentage.

\section{RESULTS}

A descriptive study was conducted among 200 subjects out of which 100 were patients and 100 caregivers. In this study, the male: female ratio was 59: 41 among diabetics and 31: 19 among caregivers. Among the diabetics $38 \%$ had average awareness about symptoms of hypoglycaemia, whereas $48 \%$ of caregivers were poorly aware. Only $40 \%$ of diabetics got their diabetic education, which was mostly from their clinicians. Regarding management of hypoglycaemia, 38\% patients told taking glucose powder or sugar with water as an immediate measure; whereas $39 \%$ caregivers opted just water. Majority of patients (60\%) and caregivers $(67 \%)$ felt immediate treatment in casualty or emergency department was the right choice for treating hypoglycaemia. The literacy level of patients (55\%) and caregiver having good awareness on symptoms of hypoglycaemia was above high school level (96\%).

\section{DISCUSSION}

This descriptive study was conducted among 200 subjects, with 100 patients and caregivers. Majority fell between the age group of 51-60 years which included 54 (54\%) diabetics and $62(62 \%)$ caregivers. Study conducted in Germany showed that older group of people showed reduced awareness of hypoglycaemia as compared to middle-aged. Thus, hypoglycaemic symptoms were more encountered among the elderly. 4

In a 12 year follow up study from San Francisco, among 783 adults with diabetes mellitus, elderly with dementia had greater risk of having hypoglycaemia when compared to others. ${ }^{5}$ Presence of dementia in elderly could contribute to frequent occurrence of hypoglycaemia, probably from accidental excessive doses of oral hypoglycaemic agents (OHA's) or insulin. Zammitt NN and Frier BM observed increased risk of hypoglycaemia among elderly and in those with longer duration of diabetes. ${ }^{6}$ In contrast, present study had elderly with more awareness on hypoglycaemia than young patients.
In the present study $61 \%$ were on OHA's, $10 \%$ on Insulin and remaining $29 \%$ were on both drugs. Among patients on OHA's 77\% had good awareness about the symptoms of hypoglycaemia. Commonest symptoms experienced were fatigue (80\%), headache (78\%), excessive sweating (70\%). A study from England among Type-2 diabetics, 5.9\% patients on OHA's in the age group of 40 to 65 years had experienced hypoglycaemic symptoms. None required hospitalisation as they aware on the immediate management of hypoglycaemia as they had good diabetic education. ${ }^{7}$ About $50 \%$ patients treated with insulin were poorly aware of the symptoms of hypoglycaemia. Schopman et al showed impaired awareness of hypoglycaemia among insulin treated diabetics and a 17fold increased incidence of symptomatic hypoglycaemia among insulin treated patients than among others. ${ }^{8}$ Leese GP et al from UK noted severe hypoglycaemia among type- 2 diabetics treated with insulin than those on OHA's. ${ }^{9}$

Patients on irregular treatment experienced hypoglycaemic symptoms more frequently when compared to patients on regular medications. The present study showed patients on insulin to have poor aware on symptoms of hypoglycaemia. Most patients on insulin tend to adjust dosage by themselves to avoid/ delay a doctor consultation. If such patients fail to recognise initial symptoms of hypoglycaemia, the chances of them developing severe hypoglycaemia was higher. Elderly with visual or motor impairment might self-administer altered doses of insulin for their difficulty in handling insulin pens or syringes. Their caregivers must be aware of the techniques and flaws of insulin administration along with symptoms of hypoglycaemia.

In the current study, $38 \%$ of diabetics had average awareness on hypoglycaemic symptoms, but $48 \%$ of caregivers had poor awareness. Among the diabetics we studied only $40 \%$ had attended diabetic education classes. The education programs or information was from their treating doctors $(82 \%)$, health-care worker $(13 \%)$ and diabetic clinics (5\%) elsewhere. Among the caregivers 74\% got diabetes education from doctor; and 13\% each from a diabetic clinic or health-care worker.

On the immediate treatment of hypoglycaemia, $38 \%$ of diabetics opined immediate need of taking glucose powder or sugar with water but $39 \%$ of caregivers opted for taking water. Majority of patients (60\%) and caregivers (67\%) felt, seeking medical help at hospital emergency department as the right option in managing hypoglycaemia. The more literate the patients were, they could identify symptoms of hypoglycaemia and take appropriate measures. The literacy level of patients (55\%) and caregivers (96\%) with good awareness on symptoms of hypoglycaemia were above high school level.

Among patients who had good awareness on symptoms of hypoglycaemia; $73 \%$ were males, $65 \%$ had $5-10$ years of diabetes and $65 \%$ had attended diabetic education classes. Among these patients only $15 \%$ had ever experienced hypoglycaemic symptoms. Ya-Chun et al studied 1195 patients with type- 2 diabetes and observed $7.4 \%$ to have experienced severe hypoglycaemia. Patients on insulin experienced hypoglycaemia more frequently $(17.8 \%)$ as compared to patients on OHA's (6.3\%). The usual risks for developing severe hypoglycaemia was older age, lower 


\section{Jemds.com}

literacy level and inconsistent dietary habits while on insulin therapy. ${ }^{10}$

\section{CONCLUSIONS}

Patients were more aware about immediate treatment for hypoglycaemia than caregivers. Hence caregivers must be educated about hypoglycaemia as they are the ones who would be taking immediate action when patient is hypoglycaemic. Awareness regarding hypoglycaemia increased with literacy.

\section{REFERENCES}

[1] Wild S, Roglic G, Green A, et al. Global prevalence of diabetes: estimates for the year 2000 and projections for 2030. Diabetes Care 2004;27(5):1047-53.

[2] Kaveeshwar SA, Cornwall J. The current state of diabetes mellitus in India. Australas Med J 2014;7(1):45-8.

[3] Ostenson CG, Geelhoed-Duijvestijn P, Lahtela J, et al. Self-reported non-severe hypoglycaemic events in Europe. Diabet Med 2014;31(1):92-101.

[4] Bremer JP, Jauch-Chara K, Hallschmid M, et al. Hypoglycaemia unawareness in older compared with middle-aged patients with type 2 diabetes. Diabetes Care 2009;32(8):1513-7.

\section{Original Research Article}

[5] Yaffe K, Falvey CM, Hamilton N, et al. Association between hypoglycaemia and dementia in a biracial cohort of older adults with diabetes mellitus. JAMA Intern Med 2013;173(14):1300-6.

[6] Zammitt NN, Frier BM. Hypoglycaemia in type 2 diabetes: pathophysiology, frequency, and effects of different treatment modalities. Diabetes Care 2005;28(12):2948-61.

[7] Jennings AM, Wilson RM, Ward JD. Symptomatic hypoglycaemia in NIDDM patients treated with oral hypoglycaemic agents. Diabetes Care 1989;12(3):2038.

[8] Schopman JE, Geddes J, Frier BM. Prevalence of impaired awareness of hypoglycaemia and frequency of hypoglycaemia in insulin-treated type-2 diabetes. Diabetes Res Clin Pract 2010;87(1):64-8.

[9] Leese GP, Wang J, Broomhall J, et al. Frequency of severe hypoglycaemia requiring emergency treatment in type 1 and type 2 diabetes: a population-based study of health service resource use. Diabetes Care 2003;26(4):1176-80.

[10] Hsiao, YC, Chien MN. Severe hypoglycaemia in type 2 diabetes a hospital-based retrospective study. J Intern Med Taiwan 2006;17:73-7. 